\title{
Patterns and predictors of statin use after coronary artery bypass graft surgery
}

\author{
Alexander Kulik, MD, ${ }^{\text {a }}$ Raisa Levin, MS, ${ }^{\text {b }}$ Marc Ruel, MD, MPH, ${ }^{\text {a }}$ Thierry G. Mesana, MD, PhD, \\ Daniel H. Solomon, MD, MPH, and Niteesh K. Choudhry, MD, PhD ${ }^{\mathrm{b}}$
}

Objectives: The benefits of statin therapy for patients with coronary artery disease have been well documented, including those occurring after coronary artery bypass graft surgery. The purposes of this study were to assess statin prescription rates in patients who have undergone coronary artery bypass graft surgery and to identify the determinants of postoperative statin administration.

Methods: A retrospective cohort of 9284 Medicare patients aged 65 years or older who underwent coronary artery bypass graft surgery (1995-2004) was assembled by using linked hospital and pharmacy claims data. Rates of statin use after hospital discharge were calculated, and predictors of postoperative statin use were identified by using generalized estimating equations.

Results: Overall, $35.9 \%$ of patients received statins within 90 days of coronary artery bypass graft surgery discharge. Use of statins within 90 days after coronary artery bypass graft surgery steadily improved during the study period, from $13.1 \%$ in 1995 to $60.9 \%$ in 2004. Patient factors independently associated with an increase in postoperative statin therapy included preoperative statin use (odds ratio, 7.69), later year of operation (odds ratio, 1.22 per additional year), and additional postoperative medications (odds ratio, 1.16 per additional medication). Factors independently associated with a decrease in postoperative statin therapy included peripheral vascular disease (odds ratio, 0.60), diabetes mellitus (odds ratio, 0.67), stroke (odds ratio, 0.77), and older age (odds ratio, 0.96 per additional year). Surgeon and hospital characteristics were not independently associated with postoperative statin use.

From the Division of Cardiac Surgery, ${ }^{\mathrm{a}}$ University of Ottawa Heart Institute, Ottawa, Canada; and the Division of Pharmacoepidemiology and Pharmacoeconomics, ${ }^{\mathrm{b}}$ Brigham and Women's Hospital, Harvard Medical School, Boston, Mass.

Daniel H. Solomon received substantial grant support from Merck and Pfizer Pharmaceutical Companies, which manufacture the statins referred to in the study.

Received for publication March 14, 2007; revisions received May 4, 2007; accepted for publication May 14, 2007.

Address for reprints: Niteesh K. Choudhry, $\mathrm{MD}, \mathrm{PhD}$, Brigham and Women's Hospital, 1620 Tremont St, Suite 3030, Boston, MA, 02120 (E-mail: nchoudhry@partners.org).

J Thorac Cardiovasc Surg 2007;134:932-8

$0022-5223 / \$ 32.00$

Copyright $\odot 2007$ by The American Association for Thoracic Surgery

doi:10.1016/j.jtcvs.2007.05.039
Conclusions: Statins are considerably underused after coronary artery bypass graft surgery, although recent prescription rates are increasing. Patterns of use do not appear to correlate with coronary artery disease risk. These findings highlight the need for targeted quality improvement initiatives to increase the rate of statin administration to this at-risk population.

A lthough coronary artery bypass graft (CABG) surgery effectively treats ischemic heart disease, the long-term results after CABG surgery are compromised by the progression of atherosclerosis in native coronary arteries and saphenous vein bypass grafts. ${ }^{1,2}$ Only $60 \%$ of vein grafts remain patent 10 years after surgical intervention, and $50 \%$ of those that are patent have clinically important stenosis. ${ }^{1,2}$ Accordingly, patients after CABG surgery are at high risk for subsequent ischemic events, including death, myocardial infarction, and stroke. ${ }^{1,2}$

Clinical trials have consistently demonstrated that statins reduce the risk of recurrent cardiovascular events and improve survival in patients with coronary artery disease (CAD), including survival in patients after CABG surgery. ${ }^{3-5}$ In the largest study involving patients after surgical revascularization, treatment with 40 to $80 \mathrm{mg}$ of lovastatin daily reduced low-density lipoprotein (LDL) cholesterol levels to less than 


\section{Abbreviations and Acronyms \\ $\mathrm{CABG}=$ coronary artery bypass grafting \\ $\mathrm{CAD}=$ coronary artery disease \\ $\mathrm{CI}=$ confidence interval \\ GEE = generalized estimating equation \\ LDL $=$ low-density lipoprotein \\ $\mathrm{OR}=$ odds ratio \\ PAAD $=$ New Jersey Pharmaceutical Assistance to the Aged and Disabled \\ PACE $=$ Pennsylvania Pharmaceutical Assistance Contract for the Elderly}

$100 \mathrm{mg} / \mathrm{dL}$ and significantly reduced the progression of postoperative vein graft disease compared with moderate cholesterol treatment with 2.5 to $5 \mathrm{mg}$ of lovastatin daily. ${ }^{5}$

The American Heart Association/American College of Cardiology Secondary Prevention Clinical Guidelines ${ }^{6}$ and the National Cholesterol Education Program Adult Treatment Panel III Guidelines ${ }^{7,8}$ currently recommend treatment to achieve LDL levels of less than $100 \mathrm{mg} / \mathrm{dL}$ for patients with documented atherosclerotic vascular disease, including patients after CABG surgery. Because less than $7 \%$ of patients with atherosclerosis are able to achieve LDL levels of less than $100 \mathrm{mg} / \mathrm{dL}$ with diet and exercise regimens $\mathrm{s}^{9,10}$ in the absence of severe contraindications, essentially all patients after CABG surgery are candidates for long-term postoperative statin therapy. ${ }^{11}$ Whether patients undergoing CABG surgery actually receive statins after the operation is largely unknown. Therefore we sought to assess the rate of statin use among patients discharged from the hospital after CABG surgery and to identify the determinants of statin use in this population.

\section{Materials and Methods \\ Setting and Design}

We assembled a retrospective cohort of Medicare patients who underwent $\mathrm{CABG}$ surgery by linking Medicare files to data from the Pennsylvania Pharmaceutical Assistance Contract for the Elderly (PACE) and the New Jersey Pharmaceutical Assistance to the Aged and Disabled (PAAD) programs. Both PACE and PAAD provide prescription drug benefits to lower middle-income individuals aged 65 years or older whose yearly earnings are above the threshold to qualify them for Medicaid. Participants pay copayments of between $\$ 5$ and $\$ 10$ per prescription without any deductibles. The programs cover all medications that require a prescription and do not restrict which medications can be prescribed (ie, the programs do not use formularies, preferred drug lists, or prior authorization programs).

Data from PACE, PAAD, and Medicare were incorporated into a relational database consisting of data for all filled prescriptions, procedures, physician encounters, hospitalizations, long-term care admissions, and deaths for the patients in this cohort. These data sources have been used extensively to study population-based health outcomes. ${ }^{12,13}$ All traceable person-specific identifying factors were transformed into anonymous, coded study numbers to protect subjects' privacy. This study was approved by the institutional review board of the Brigham and Women's Hospital.

\section{Cohort}

We included patients who were discharged from the hospital after undergoing CABG surgery (International Classification of Diseases-Ninth Revision $36.1 \mathrm{x}$ or $36.2 \mathrm{x}$ ) between January 1, 1995, and December 31, 2004. We excluded patients who died within 90 days after surgical intervention, patients who were not active users of either drug benefit program, and patients who received prescriptions for cerivastatin since this drug was withdrawn from the market. The date of discharge from the hospital after CABG was considered as the index date for the study analysis.

\section{Statin Use}

We assessed statin prescription rates (ie, prescriptions that were filled) in the 1-year period before CABG surgery, as well as within 90 days, 180 days, and 365 days after the CABG discharge date. Patients were classified into drug and dose categories based on their statin prescription data before and after surgical intervention. "High-intensity statins" were those that would be expected to decrease LDL cholesterol levels by greater than $40 \%$ (atorvastatin, $>10 \mathrm{mg}$; lovastatin, $>40 \mathrm{mg}$; rosuvastatin, $>5 \mathrm{mg}$; simvastatin, $>40 \mathrm{mg}$ ). All other statin drug-dose combinations were considered "low-intensity statins" (atorvastatin, $\leq 10 \mathrm{mg}$; lovastatin, $\leq 40 \mathrm{mg}$; rosuvastatin, $\leq 5 \mathrm{mg}$; simvastatin, ' $40 \mathrm{mg}$; any dose of fluvastatin; any dose of pravastatin). ${ }^{8,14}$

\section{Patient Covariates}

We determined patient comorbidities by searching physician service claims and hospitalization records for relevant diagnostic codes in the 1-year period before the index date. In this manner the following characteristics were identified: age at index date, year of operation, sex, race, length of hospital stay, previous myocardial infarction or acute coronary syndrome, hypertension, diabetes mellitus, congestive heart failure, stroke, peripheral vascular disease, previous $\mathrm{CABG}$ surgery, previous percutaneous coronary intervention, chronic kidney disease, and chronic obstructive pulmonary disease. We also determined the use of the following concurrent medications in the 1-year period before and 90 days after CABG surgery: angiotensin-converting enzyme inhibitors or angiotensin II receptor blockers, $\beta$-blockers, calcium-channel blockers, fibrates, diuretics, nitrates, digoxin, warfarin, and clopidogrel.

\section{Hospital and Surgeon Covariates}

We identified the hospital and surgeon for each CABG procedure. Hospitals that were accredited with the Association of American Medical Colleges were classified as teaching hospitals. All other hospitals were classified as nonteaching hospitals. We classified the "operating surgeon" as the cardiac, cardiothoracic, or thoracic surgeon who submitted a claim for CABG on the date of surgical intervention using Medicare Part B claims. Records containing invalid provider identification numbers were excluded from further analysis. If 2 or more surgeons were identified for an individual patient, then we defined the most responsible surgeon as the 
surgeon that submitted the most claims for that specific patient after surgical intervention. The annualized volume of Medicare patients undergoing CABG surgery treated by each surgeon was estimated by dividing the total number of Medicare patients undergoing CABG surgery for each surgeon during the study time period by the number of years that each surgeon treated 1 or more patients. Surgeons were ranked in order of annualized volume and were then categorized into high-, medium-, and low-volume surgeon tertiles of equal size. The annual volume of the hospital from which each patient was discharged was determined in a similar manner as for surgeon volume, and high-, medium-, and lowvolume hospital tertiles were subsequently created.

\section{Statistical Analysis}

Our primary outcome was the rate of statin prescriptions that were filled within 90 days after CABG discharge. We compared statin users and nonusers using unpaired 2-sided Student $t$ tests, Fisher exact tests, or $\chi^{2}$ trend tests, as appropriate. Independent predictors of postoperative statin use within 90 days after CABG discharge were identified by using generalized estimating equations (GEEs) with clustering at the surgeon level. Factors of clinical relevance thought to affect postoperative statin rates were incorporated into the GEE analysis, including age, sex, race, year of operation, number of postoperative medications, peripheral vascular disease, preoperative stroke, recent myocardial infarction or acute coronary syndrome, hypertension, diabetes mellitus, preoperative statin use, teaching hospital, hospital volume, and surgeon volume. Odds ratios (ORs) are presented with $95 \%$ confidence intervals (CIs). All analyses were performed with SAS version 8.2 software (SAS Institute, Cary, NC).

\section{Sensitivity Analysis}

Additional analyses were performed for confirmatory purposes, including the use of interaction terms in the GEE analyses and the removal of patients from the cohort who required long-term care after surgical intervention. Other methods of categorization (instead of tertiles) were used to further evaluate associations between surgeon and hospital volume and postoperative statin use.

\section{Results}

\section{Patient Cohort}

Our cohort consisted of 9284 patients who underwent CABG surgery between 1995 and 2004. The mean age of the cohort was $75.7 \pm 5.5$ years, and $64.3 \%$ of patients were women. Preoperatively, the incidence of diabetes mellitus was $48.1 \%$, the incidence of stroke was $15.7 \%$, and the incidence of peripheral vascular disease was $6.0 \%$. The majority of patients underwent $\mathrm{CABG}$ surgery at teaching hospitals $(77.3 \%)$, with $89.7 \%$ undergoing operations at high-volume hospitals and $80.5 \%$ undergoing operations by high-volume surgeons. The mean length of stay after CABG surgery was $11.1 \pm 7.2$ days.

\section{Statin Prescription Rates}

During the 1-year period before CABG surgery, $37.3 \%$ of patients received a statin prescription (low-intensity statins,

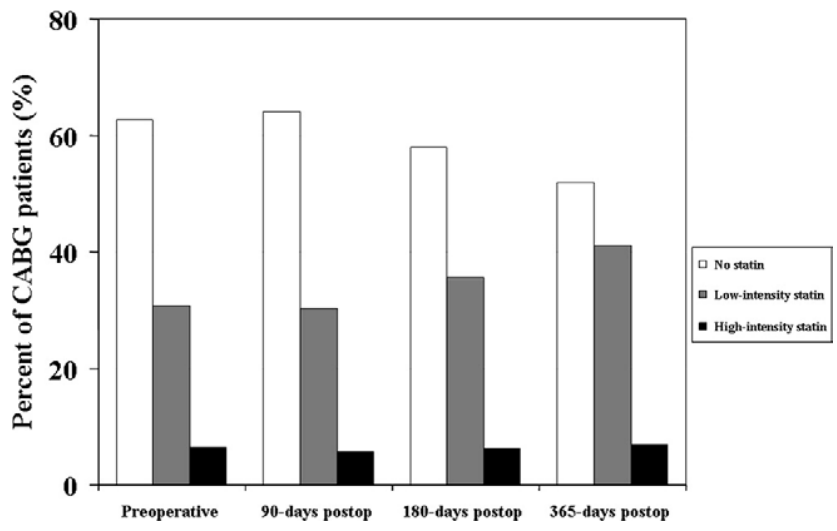

Figure 1. Statin prescription rates before and after coronary artery bypass grafting (CABG) surgery. Prescription rates decreased slightly from before surgical intervention to $\mathbf{9 0}$ days after surgical intervention but then steadily increased to 1 year after CABG surgery.

30.8\%; high-intensity statins, 6.5\%). In contrast, only $35.9 \%$ of patients undergoing $\mathrm{CABG}$ surgery were prescribed statins within 90 days after the CABG procedure (low-intensity statins, 30.3\%; high-intensity statins, 5.6\%). Of the patients who received preoperative statin therapy, only $67.4 \%$ of these patients received postoperative statins. Statin administration rates were low among all patient subgroups, including those with diabetes mellitus, cerebrovascular disease, or peripheral vascular disease (statin rates of $37.5 \%, 33.1 \%$, and $32.3 \%$, respectively). In comparison with statin use, $57.3 \%$ of patients received $\beta$-blockers after surgical intervention, $36.3 \%$ received angiotensin-converting enzyme inhibitors or angiotensin II receptor blockers, $21.1 \%$ received calcium-channel blockers, $19.3 \%$ received digoxin, $17.8 \%$ received nitrates, $14.8 \%$ received warfarin, $8.0 \%$ received clopidogrel, $5.0 \%$ received diuretics, and $1.6 \%$ received fibrate therapy.

Statin prescription rates increased with duration of time after surgical intervention, from $35.9 \%$ to $42.0 \%$ to $48.1 \%$ within 90, 180, and 365 days after CABG discharge, respectively (Figure 1). Statin prescription rates also increased substantially with each additional year in the study, with a low of $13.1 \%$ in 1995 to a maximum of $60.9 \%$ in 2004 (Figure 2). The most common statins used after surgical intervention included simvastatin $(15.7 \%)$ and atorvastatin $(12.7 \%)$. Fewer patients were prescribed pravastatin $(4.6 \%)$, lovastatin $(2.0 \%)$, fluvastatin $(0.9 \%)$, and rosuvastatin $(0.1 \%)$.

\section{Predictors of Statin Use}

Table 1 describes the characteristics of the patients who did and did not receive statins within 90 days after $\mathrm{CABG}$ discharge. Statin users were more likely to have received 


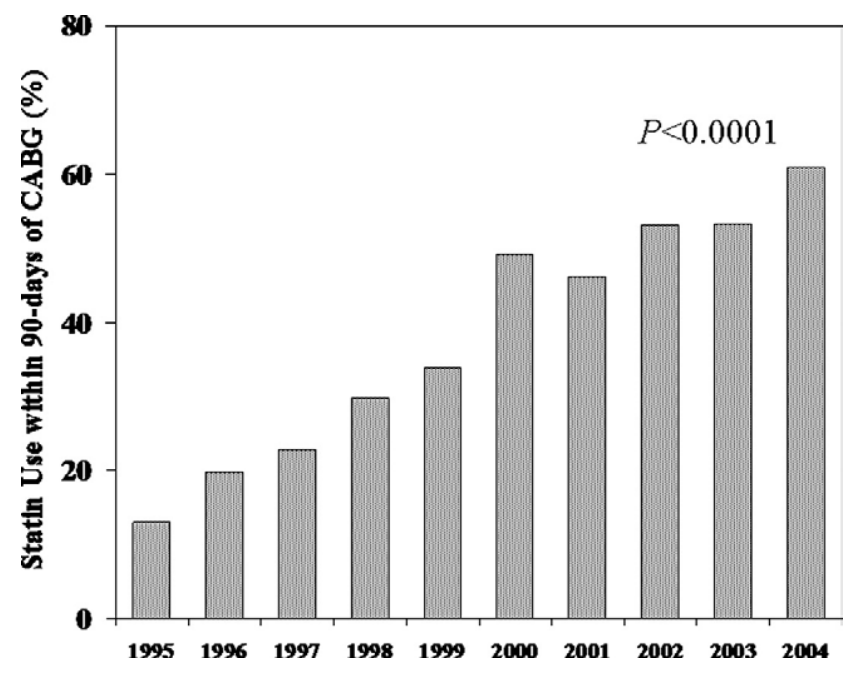

Figure 2. Statin prescription rates within $\mathbf{9 0}$ days after coronary artery bypass grafting (CABG) surgery stratified by year of operation. Statin prescription rates steadily increased each year of the study.

preoperative statins than nonusers $(P<.0001)$. Postoperative statin users were also more likely to have taken other cardiac medications both before and after surgical intervention (all $P<.05$ ). Although statin users were more likely to be female and have diabetes mellitus, nonstatin users were more likely to be older, have longer hospital stays, and have other comorbidities (stroke, congestive heart failure, and chronic obstructive pulmonary disease; all $P<.05$ ). Statin users were more likely to have had operations in recent years, although nonstatin users were more likely to have had operations at teaching hospitals $(P=.10)$ and at highvolume hospitals $(P=.01)$.

After adjusting for patient-, surgeon-, and hospital-related characteristics, the strongest predictor of postoperative statin therapy was preoperative statin use (OR, 7.69; 95\% CI, 6.81-8.68; $P<.0001$; Table 2). Later year of operation (OR, 1.22 per additional year; 95\% CI, 1.19-1.25; $P<$ $.0001)$ and additional postoperative medications (OR, 1.16 per additional medication; 95\% CI, 1.15-1.18; $P<.0001$ ) also independently predicted postoperative statin use. Factors associated with a reduction in postoperative statin use included a history of peripheral vascular disease (OR, 0.60; 95\% CI, 0.48-0.75; $P<.0001$ ), diabetes mellitus (OR, $0.67 ; 95 \%$ CI, $0.59-0.75 ; P<.0001)$, preoperative stroke (OR, 0.77; 95\% CI, 0.67-0.90; $P=.0006$ ), and older age (OR, 0.96 per additional year; 95\% CI, 0.95-0.97; $P<$ $.0001)$. Surgeon and hospital characteristics were not independently associated with postoperative statin use. Sensitivity analyses yielded nearly identical results to those presented.
TABLE 1. Baseline characteristics for patients treated with and without statins 90 days after CABG discharge

\begin{tabular}{|c|c|c|}
\hline Characteristic & $\begin{array}{l}\text { Nonstatin users } \\
(\mathrm{n}=7121)\end{array}$ & $\begin{array}{l}\text { Statin users } \\
(\mathrm{n}=3474)\end{array}$ \\
\hline \multicolumn{3}{|l|}{ Demographics } \\
\hline Age, mean $\pm S D$ & $75.9 \pm 5.5$ & $75.2 \pm 5.4^{*}$ \\
\hline Female sex, $\%$ & 62.4 & $67.8^{*}$ \\
\hline White race, $\%$ & 92.6 & 91.8 \\
\hline \multicolumn{3}{|l|}{ Comorbid conditions, $\%$} \\
\hline Prior myocardial infarction & 16.0 & $13.4^{*}$ \\
\hline Congestive heart failure & 52.6 & $47.4^{*}$ \\
\hline Stroke & 16.4 & $14.4^{*}$ \\
\hline Peripheral vascular disease & 6.4 & 5.4 \\
\hline Hypertension & 87.6 & $91.9 *$ \\
\hline Diabetes & 46.9 & $50.2^{*}$ \\
\hline Chronic kidney disease & 27.2 & 27.2 \\
\hline $\begin{array}{l}\text { Chronic obstructive pulmonary } \\
\text { disease }\end{array}$ & 32.5 & $28.8^{*}$ \\
\hline Previous CABG & 0.1 & $0.4^{*}$ \\
\hline Previous stent/PTCA & 9.0 & 9.9 \\
\hline \multicolumn{3}{|l|}{ Preoperative medication use, $\%$} \\
\hline Prior statin & 19.0 & $70.0^{*}$ \\
\hline $\begin{array}{l}\text { ACE inhibitor or angiotensin II } \\
\text { receptor blocker }\end{array}$ & 35.7 & $51.8^{*}$ \\
\hline Clopidogrel & 8.2 & $19.4^{*}$ \\
\hline$\beta$-Blocker & 47.8 & $71.6^{*}$ \\
\hline Calcium-channel blocker & 43.8 & $50.5^{*}$ \\
\hline Digoxin & 13.8 & $16.8^{*}$ \\
\hline Diuretics & 6.5 & $10.1^{*}$ \\
\hline Fibrate & 3.4 & 4.0 \\
\hline Nitrates & 47.6 & $59.5^{*}$ \\
\hline Warfarin & 10.2 & $11.7^{*}$ \\
\hline \multicolumn{3}{|l|}{ Postoperative medication use, $\%$} \\
\hline $\begin{array}{l}\text { ACE inhibitor or angiotensin II } \\
\text { receptor blocker }\end{array}$ & 29.4 & $48.6^{*}$ \\
\hline Clopidogrel & 4.1 & $14.7^{*}$ \\
\hline$\beta$-Blocker & 46.0 & $77.2^{*}$ \\
\hline Calcium-channel blocker & 18.8 & $25.0^{*}$ \\
\hline Digoxin & 17.8 & $21.9^{*}$ \\
\hline Diuretics & 3.7 & $7.3^{*}$ \\
\hline Fibrate & 1.6 & 1.5 \\
\hline Nitrates & 16.4 & $20.4^{*}$ \\
\hline Warfarin & 14.3 & 15.6 \\
\hline \multicolumn{3}{|l|}{ Hospital characteristics } \\
\hline Teaching hospital, \% & 77.9 & 76.4 \\
\hline \multicolumn{3}{|l|}{ Annual volume of hospital, \% } \\
\hline High ( $\geq 54$ patients/y) & 90.2 & 88.8 \\
\hline Medium (3-53 patients/y) & 9.2 & $10.8^{*}$ \\
\hline Low ( $\leq 2$ patients/y) & 0.7 & 0.4 \\
\hline \multicolumn{3}{|l|}{ Annual volume of surgeon, $\%$} \\
\hline High ( $\geq 20$ patients/y) & 80.5 & 80.4 \\
\hline Medium (3-19 patients/y) & 17.1 & 17.5 \\
\hline Low ( $\leq 3$ patients/y) & 2.3 & 2.1 \\
\hline Length of stay, mean $\pm S D$ & $11.8 \pm 7.2$ & $10.1 \pm 5.0^{*}$ \\
\hline
\end{tabular}

CABG, Coronary artery bypass grafting; $S D$, standard deviation; PTCA percutaneous transluminal coronary angioplasty; $A C E$, angiotensinconverting enzyme. ${ }^{*} P<.05$. 
TABLE 2. Multivariable predictors of postoperative statin use

\begin{tabular}{lc}
\hline Characteristic & $\begin{array}{c}\text { Adjusted odds ratio } \\
\text { (95\% confidence interval) }\end{array}$ \\
\hline Increased statin use & \\
Preoperative statin use & $7.69(6.81-8.68)$ \\
Year of operation (per additional & $1.22(1.19-1.25)$ \\
$\quad$ year) & \\
Postoperative medications (per & $1.16(1.15-1.18)$ \\
$\quad$ additional medication) & \\
Decreased statin use & \\
Peripheral vascular disease & $0.60(0.48-0.75)$ \\
Diabetes mellitus & $0.67(0.59-0.75)$ \\
Stroke & $0.77(0.67-0.90)$ \\
Patient age (per additional year) & $0.96(0.95-0.97)$ \\
Nonsignificant factors & \\
Male sex & $1.05(0.95-1.16)$ \\
White race & $0.99(0.82-1.20)$ \\
Prior myocardial infarction & $0.98(0.84-1.13)$ \\
Hypertension & $1.00(0.83-1.20)$ \\
Teaching hospital & $1.01(0.86-1.19)$ \\
Medium-volume vs low-volume & $1.49(0.78-2.87)$ \\
$\quad$ hospital & \\
High-volume vs low-volume hospital & $1.52(0.79-2.91)$ \\
Medium-volume vs low-volume & $0.93(0.62-1.38)$ \\
$\quad$ surgeon & \\
High-volume vs low-volume surgeon & $0.98(0.67-1.44)$ \\
\hline
\end{tabular}

\section{Discussion}

Clinical trials have consistently demonstrated that statins reduce the risk of recurrent cardiovascular events and improve survival across a wide range of cholesterol levels in patients with $\mathrm{CAD} .{ }^{3}$ The benefits of statin treatment appear to be applicable to men and women, as well as to older and younger patients. ${ }^{3}$ As one of the safest classes of drugs ever developed, ${ }^{15}$ statins are recommended as preventive therapy for patients with $\mathrm{CAD}$, even after surgical revascularization. ${ }^{7,16}$ Given the weight of the evidence and the fact that statins have been shown to reduce saphenous vein graft disease, ${ }^{4,5,17}$ in the absence of serious contraindications, effectively all patients after CABG surgery should be prescribed long-term statin therapy. ${ }^{6-8}$ In contrast, we observed that (1) overall, only $37.3 \%$ of patients received statin therapy before CABG surgery, and even fewer patients (35.9\%) were prescribed statins within 90 days postoperatively; (2) although rates of statin prescriptions have increased substantially over time, they still remain suboptimal; and (3) patients with risk factors for future vascular events, such as diabetes mellitus and history of stroke, were the least likely to receive statins.

As the largest study to date to have evaluated medical therapy after CABG surgery, the results of this research confirm those of smaller cohort studies that have docu- mented low statin prescription rates after CABG surgery. ${ }^{18,19}$ Most recently, in a study of 320 patients undergoing CABG surgery, the use of antilipid agents was $55 \%$ at admission, $57 \%$ at CABG discharge, and $76 \%$ at 12 months after surgical intervention. ${ }^{20}$ Likewise, in a study of evidencebased preventive therapies in 31,750 Duke University patients, Newby and colleagues ${ }^{21}$ reported the consistent use of lipidlowering therapy in only $44 \%$ of patients with CAD (treated with medical, percutaneous, or surgical therapy). Similar to the current study, Newby and colleagues ${ }^{21}$ also reported that the use of lipid-lowering agents increased over time, with $63 \%$ of patients taking lipid-lowering therapy in $2002 \mathrm{com}-$ pared with approximately $25 \%$ in 1995 . The results our cohort and that from Duke ${ }^{21}$ both identified older age as a predictor of lower statin use, despite the fact that the elderly derive more absolute benefit from statins as a consequence of their high baseline risk of cardiovascular disease. ${ }^{3,22}$

In addition to advanced age, patients with other cardiac risk factors paradoxically received less preventive statin therapy after surgical intervention compared with that received by patients without these risk factors. A history of peripheral vascular disease, diabetes mellitus, and stroke were all independently associated with a reduction in postoperative statin prescription rates. Patients with these risk factors could benefit the most from statin therapy. ${ }^{3,21}$ This finding is analogous to the results reported by Lee and associate $^{23}$ regarding the underuse of angiotensin-converting enzyme inhibitors and $\beta$-blockers in patients with heart failure at the greatest risk of death. Physician belief that patients with comorbidities will experience less benefit and greater harm from statin therapy is one potential explanation for this treatment-risk paradox. Alternatively, physicians might be less attentive to preventive therapy when caring for patients with multiple concurrent conditions, or they might be less inclined to prescribe medications to patients thought unlikely to adhere to treatment. ${ }^{23,24}$

The strongest factor associated with the administration of postoperative statin therapy in our cohort was the use of preoperative statins. As a consequence, encouraging appropriate preoperative statin use might be an effective strategy to increase postoperative use. Moreover, preoperative statin use reduces the incidence of adverse cardiovascular events after CABG syurgery. ${ }^{25,26}$ Still, only $67 \%$ of patients who received preoperative statins were prescribed statins after surgical intervention in this study, suggesting that preventive statin therapy is being forgotten or disregarded after CABG surgery.

Overall, too few patients are being prescribed statins after CABG surgery, and this study highlights the need for targeted quality improvement initiatives focusing on the prescription habits of cardiac surgeons and cardiologists. Several researchers have explored approaches to improving these treatment gaps in cardiovascular care and preven- 
tion. ${ }^{22,27}$ Audit with feedback on performance is one important method that can be used to achieve this goal among patients after CABG surgery and should be explored further. ${ }^{20}$ Moreover, recent randomized controlled trials have demonstrated that, compared with usual care, the addition of electronic information systems (electronic medical record reminders and automated voice message reminders) or the use of integrated team care models (pharmacy team outreach groups and transition coaches) might improve medication administration and therapeutic monitoring after hospital discharge. $^{28,29}$

Through the use of a large database and robust statistical methods, we believe that our study provides the best current evaluation of statin administration after CABG surgery. However, our results should be interpreted in the context of several limitations.

First, this research focused specifically on elderly patients enrolled in Medicare and the PACE and PAAD prescription drug benefit plans. Because the average age of patients undergoing CABG surgery in this cohort was 75.7 years and $64.7 \%$ of patients were female, our results might not be generalizable to patients with other demographic or clinical characteristics.

Second, the administrative data used does not contain detailed clinical information, such as cholesterol levels, or the reasons for physicians' prescription choices. For example, statins might have preferentially been prescribed to patients with higher baseline cholesterol levels. Moreover, it is possible that patients who filled their statin prescriptions in this study might not have actually ingested the medications. That said, it is generally agreed that the rate of prescriptions being filled by patients well approximates their actual consumption. ${ }^{30}$

Finally, it is impossible to differentiate patient noncompliance in filling a prescription from physician nonprescription in our data.

In summary, much progress has been made in the evaluation of preventive therapies, and yet many patients do not receive the medical therapies that can improve their outcomes. This study illustrates that statins are considerably underused after CABG surgery. However, their use has increased in recent years. These findings highlight the need for targeted quality improvement initiatives to further increase the rate of statin administration to this at-risk population.

\section{References}

1. Fitzgibbon GM, Kafka HP, Leach AJ, Keon WJ, Hooper GD, Burton JR. Coronary bypass graft fate and patient outcome: angiographic follow-up of 5,065 grafts related to survival and reoperation in 1,388 patients during 25 years. J Am Coll Cardiol. 1996;28:616-26.

2. Motwani JG, Topol EJ. Aortocoronary saphenous vein graft disease: pathogenesis, predisposition, and prevention. Circulation. 1998;97: 916-31.
3. Baigent C, Keech A, Kearney PM, Blackwell L, Buck G, Pollicino C, et al. Efficacy and safety of cholesterol-lowering treatment: prospective meta-analysis of data from 90,056 participants in 14 randomised trials of statins. Lancet. 2005;366:1267-78.

4. Yang Z, Kozai T, van der Loo B, Viswambharan H, Lachat M, Turina MI, et al. HMG-CoA reductase inhibition improves endothelial cell function and inhibits smooth muscle cell proliferation in human saphenous veins. J Am Coll Cardiol. 2000;36:1691-7.

5. The Post Coronary Artery Bypass Graft Trial Investigators. The effect of aggressive lowering of low-density lipoprotein cholesterol levels and low-dose anticoagulation on obstructive changes in saphenous-vein coronary-artery bypass grafts. N Engl J Med. 1997; 336:153-62.

6. Smith SC Jr, Allen J, Blair SN, Bonow RO, Brass LM, Fonarow GC, et al. AHA/ACC guidelines for secondary prevention for patients with coronary and other atherosclerotic vascular disease: 2006 update: endorsed by the National Heart, Lung, and Blood Institute. Circulation. 2006;113:2363-72.

7. Executive summary of the Third Report of The National Cholesterol Education Program (NCEP) Expert Panel on Detection, Evaluation, And Treatment of High Blood Cholesterol In Adults (Adult Treatment Panel III). JAMA. 2001;285:2486-97.

8. Grundy SM, Cleeman JI, Merz CN, Brewer HB Jr, Clark LT, Hunninghake $\mathrm{DB}$, et al. Implications of recent clinical trials for the National Cholesterol Education Program Adult Treatment Panel III guidelines. Circulation. 2004;110:227-39.

9. Sueta CA, Chowdhury M, Boccuzzi SJ, Smith SC Jr, Alexander CM, Londhe A, et al. Analysis of the degree of undertreatment of hyperlipidemia and congestive heart failure secondary to coronary artery disease. Am J Cardiol. 1999;83:1303-7.

10. Pearson TA, Laurora I, Chu H, Kafonek S. The lipid treatment assessment project (L-TAP): a multicenter survey to evaluate the percentages of dyslipidemic patients receiving lipid-lowering therapy and achieving low-density lipoprotein cholesterol goals. Arch Intern Med. 2000; 160:459-67.

11. Lazar HL. Role of statin therapy in the coronary bypass patient. Ann Thorac Surg. 2004;78:730-40.

12. Wang PS, Schneeweiss S, Avorn J, Fischer MA, Mogun H, Solomon $\mathrm{DH}$, et al. Risk of death in elderly users of conventional vs. atypical antipsychotic medications. $N$ Engl J Med. 2005;353:2335-41.

13. Solomon DH, Schneeweiss S, Glynn RJ, Kiyota Y, Levin R, Mogun H, et al. Relationship between selective cyclooxygenase-2 inhibitors and acute myocardial infarction in older adults. Circulation. 2004;109: 2068-73.

14. Law MR, Wald NJ, Rudnicka AR. Quantifying effect of statins on low density lipoprotein cholesterol, ischaemic heart disease, and stroke: systematic review and meta-analysis. BMJ. 2003;326:1423.

15. Black DM. Gut-acting drugs for lowering cholesterol. Curr Atheroscler Rep. 2002;4:71-5.

16. Smith SC Jr, Blair SN, Bonow RO, Brass LM, Cerqueira MD, Dracup $\mathrm{K}$, et al. AHA/ACC scientific statement: AHA/ACC guidelines for preventing heart attack and death in patients with atherosclerotic cardiovascular disease: 2001 update: a statement for healthcare professionals from the American Heart Association and the American College of Cardiology. Circulation. 2001;104:1577-9.

17. Blankenhorn DH, Nessim SA, Johnson RL, Sanmarco ME, Azen SP, Cashin-Hemphill L. Beneficial effects of combined colestipol-niacin therapy on coronary atherosclerosis and coronary venous bypass grafts. JAMA. 1987;257:3233-40.

18. Belcher PR, Gaw A, Cooper M, Brown M, Wheatley DJ, Lindsay GM. Are we negating the benefits of CABG by forgetting secondary prevention? J Hum Hypertens. 2002;16:691-7.

19. Bradshaw PJ, Jamrozik K, Gilfillan I, Thompson PL. Preventing recurrent events long term after coronary artery bypass graft: suboptimal use of medications in a population study. Am Heart J. 2004; 147:1047-53.

20. Okrainec K, Pilote L, Platt R, Eisenberg MJ. Use of cardiovascular medical therapy among patients undergoing coronary artery bypass graft surgery: results from the ROSETTA-CABG registry. Can J Cardiol. 2006;22:841-7. 
21. Newby LK, LaPointe NM, Chen AY, Kramer JM, Hammill BG, DeLong ER, et al. Long-term adherence to evidence-based secondary prevention therapies in coronary artery disease. Circulation. 2006;113: 203-12.

22. McAlister FA, Taylor L, Teo KK, Tsuyuki RT, Ackman ML, Yim R, et al. The treatment and prevention of coronary heart disease in Canada: do older patients receive efficacious therapies? The Clinical Quality Improvement Network (CQIN) Investigators. J Am Geriatr Soc. 1999;47:811-8.

23. Lee DS, Tu JV, Juurlink DN, Alter DA, Ko DT, Austin PC, et al. Risk-treatment mismatch in the pharmacotherapy of heart failure. JAMA. 2005;294:1240-7.

24. Ko DT, Mamdani M, Alter DA. Lipid-lowering therapy with statins in high-risk elderly patients: the treatment-risk paradox. JAMA. 2004; 291:1864-70.

25. Dotani MI, Elnicki DM, Jain AC, Gibson CM. Effect of preoperative statin therapy and cardiac outcomes after coronary artery bypass grafting. Am J Cardiol. 2000;86:1128-30, A6.
26. Pan W, Pintar T, Anton J, Lee VV, Vaughn WK, Collard CD. Statins are associated with a reduced incidence of perioperative mortality after coronary artery bypass graft surgery. Circulation. 2004;110(suppl): II45-9.

27. Montague T, Taylor L, Martin S, Barnes M, Ackman M, Tsuyuki R, et al. Can practice patterns and outcomes be successfully altered? Examples from cardiovascular medicine. The Clinical Quality Improvement Network (CQIN) Investigators. Can J Cardiol. 1995;11: 487-92.

28. Feldstein AC, Smith DH, Perrin N, Yang X, Rix M, Raebel MA, et al Improved therapeutic monitoring with several interventions: a randomized trial. Arch Intern Med. 2006;166:1848-54.

29. Coleman EA, Parry C, Chalmers S, Min SJ. The care transitions intervention: results of a randomized controlled trial. Arch Intern Med. 2006; $166: 1822-8$

30. Schneeweiss S, Avorn J. A review of uses of health care utilization databases for epidemiologic research on therapeutics. J Clin Epidemiol. 2005;58:323-37. 